\title{
Invasive Fungal Rhinosinusitis: Our Experience
}

\author{
${ }^{1}$ Chopra H, ${ }^{2}$ Dua Kapil, ${ }^{3}$ Chopra Neha, ${ }^{4}$ Puri Sanjeev, ${ }^{5}$ Mittal Vikrant \\ ${ }^{1}$ Professor and Head, Department of ENT, Dayanand Medical College, Ludhiana, Punjab, India \\ ${ }^{2}$ Associate Professor, Department of ENT, Dayanand Medical College, Ludhiana, Punjab, India \\ ${ }^{3}$ Intern, Department of ENT, Dayanand Medical College, Ludhiana, Punjab, India \\ ${ }^{4,5}$ Senior Resident, Department of ENT, Dayanand Medical College, Ludhiana, Punjab, India
}

Correspondence: Chopra H, Professor and Head, Department of ENT, Dayanand Medical College, Ludhiana, Punjab, India

\begin{abstract}
Opportunistic fungal infections in immunocompromised patients are associated with a high mortality rate. Endemic mycoses are often asymptomatic, but in appropriate hosts, fungi can cause severe and even fatal infection. Facial pain in an immunocompromised patient may signify invasive fungal sinusitis. Treatment with antifungal agents needs to be individualized according to factors such as the type of fungus, presence of renal failure, or pregnancy. Combining antifungal agents or addition of other approaches, such as surgical debridement or steps to control intracranial pressure, may be needed for adequate treatment of certain types of fungal infections.
\end{abstract}

Keywords: Immunocompromised, mucormycosis, aspergillosis.

\section{INTRODUCTION}

Acute or fulminant fungal sinusitis usually affects diabetics and patients in immunosuppressive states secondary to chemotherapy, hematologic disorders, transplantation, and AIDS also place their hosts at risk for opportunistic infection. The offending fungi usually originate from the classes zygomycetes (Mucor spp) and Ascomycetes (Aspergillus spp). Within the Mucoraceae family are the genera Rhizopus, Mucor, and Absidia with species of Rhizopus being responsible for the most serious infections. Aspergillus species include A fumigatus, A flavus, A niger, and A oryzae with $A$ fumigatus being most commonly responsible for Aspergillus.

In immunocompromised patients, Mucor spores settle onto the mucosa of nose and PNS. They penetrate into the tissue, allowing angioinvasion to occur. Mucor has a predilection for the internal elastic lamina of the arteries. The invasion produces thrombosis, with secondary ischemic infarction and hemorrhagic necrosis. The fungus thrives in this environment and spreads along injured vessels. Angioinvasion may also occur with Aspergillus, resulting in a mycotic aneurysm or thrombosis formation.

Histologic examination reveals necrosis of the tissue, neutrophilic infiltration, and hyphae. The Mucor hyphae are nonseptate with branching near 90 degrees in contrast to Aspergillus hyphae that are septate with dichotomous branching. Radiographic evaluation with CT and MRI are useful in assessing the extent of invasive fungal sinusitis. CT better defines soft tissue invasion, necrosis, and early bone erosion. MRI best evaluates early changes in major vessels, including carotid artery thrombosis, cavernous sinus thrombosis, and intracranial extension. Cavernous sinus thrombosis is well-delineated by both MRI and CT.

In addition to the prompt and accurate diagnosis, the underlying medical problem needs to be addressed because it confers important prognostic implications. Diabetic patients have an overall survival rate of $60 \%$, compared with $70 \%$ in patients with no underlying disease and $20 \%$ in patients with other systemic disorders, demonstrating the morbidity and mortality that can be associated with the fungal infection of the underlying disease.

Once diagnosed, both medical and surgical intervention is the treatment of choice. All devitalized tissues need debridement to prevent the fungus from proliferating in the necrotic tissue. Re-establishing the vitalized and bleeding tissue will allow pharmacologic agents to reach the necessary areas. The patients are put on Amphotericin B/ Liposomal Amphotericin or Voriconazole depending upon the type of disease and tolerance of the patient.

\section{PATIENTS AND METHODS}

A series of 36 patients with suspected invasive fungal rhinosinusitis were taken up for the study, preoperative investigations included diagnostic nasal endoscopy, CT scan of the nose and paranasal sinuses, nasal smear study for fungus. All these patients underwent endoscopic debridement of the disease. Postoperative confirmation of diagnosis was made by histopathology, fungal smears with 
$\mathrm{KOH}$. All these patients were treated with I/V and/ or oral anti-fungal therapy and followed up for 6 months after surgery with diagnostic nasal endoscopy.

\section{OBSERVATION AND RESULTS}

The case study comprised of 24 male and 12 female patients, with $36 \%$ in $0-35$ years age group and $64 \%$ in $36-70$ years age group (Table 1). The major presenting symptom in our study was nasal blockade (69\%). Along with this headache was seen in $58 \%$ and eye involvement in (56\%) of the patients (Table 2 and Fig. 1).

Table 1: Age and sex distribution

$\begin{array}{lccc}\text { Age } & \text { No } & \text { Males } & \text { Females } \\ \text { 0-35 years } & \text { 13(36\%) } & 10 & 3 \\ \text { 36-70 years } & \text { 23(64\%) } & 14 & 9\end{array}$

Table 2: Clinical presentation

$\begin{array}{lr}\text { Clinical presentation } & \text { No } \\ \text { Facial pain/headache } & 21(58 \%) \\ \text { Chemosis } & 24(67 \%) \\ \text { Proptosis } & 23(64 \%) \\ \text { Ophthalmoplegia } & 20(56 \%) \\ \text { Loss of vision } & 8(22 \%) \\ \text { Diplopia } & 14(39 \%) \\ \text { Nasal blockade } & 25(69 \%) \\ \text { Intracranial extension } & 8(22 \%) \\ \text { Palatal involvement } & 8(22 \%)\end{array}$

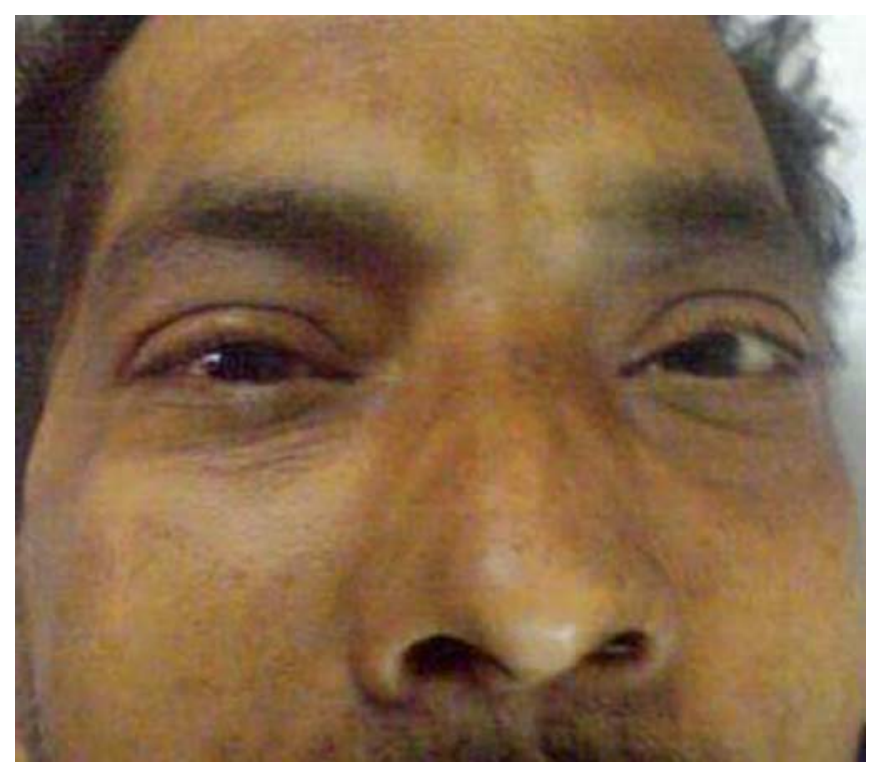

Figure 1: Clinical picture of patient showing right orbital proptosis

\section{INVESTIGATIONS}

\section{Diagnostic nasal endoscopy}

\begin{tabular}{lr}
\hline DNS & $17(47 \%)$ \\
Boggy uncinate process & $12(33 \%)$ \\
Concha bullosa & $8(22 \%)$ \\
Purulent secretions in middle meatus & $26(72 \%)$ \\
Blackish crusting & $19(53 \%)$ \\
Polypoidal changes & $17(47 \%)$ \\
Cheesy debris & $15(42 \%)$ \\
\hline
\end{tabular}

In diagnostic nasal endoscopy blackish crusting was seen in $53 \%$ of cases and $72 \%$ cases showing purulent secretions in middle meatus.

\section{CT Nose and PNS}

\begin{tabular}{lc}
\hline DNS & $20(56 \%)$ \\
Concha bullosa & $10(28 \%)$ \\
Eroded lamina papyracea & $26(72 \%)$ \\
Eroded ethmoidal septae & $18(50 \%)$ \\
Orbital involvement & $22(61 \%)$ \\
Intracranial extension & $9(25 \%)$ \\
Hyperdense shadows & $14(39 \%)$ \\
\hline
\end{tabular}

In CT Nose and PNS findings, $72 \%$ of the patients showed eroded lamina papyracea followed by orbital involvement in $61 \%$ of cases (Figs 2 and 3).

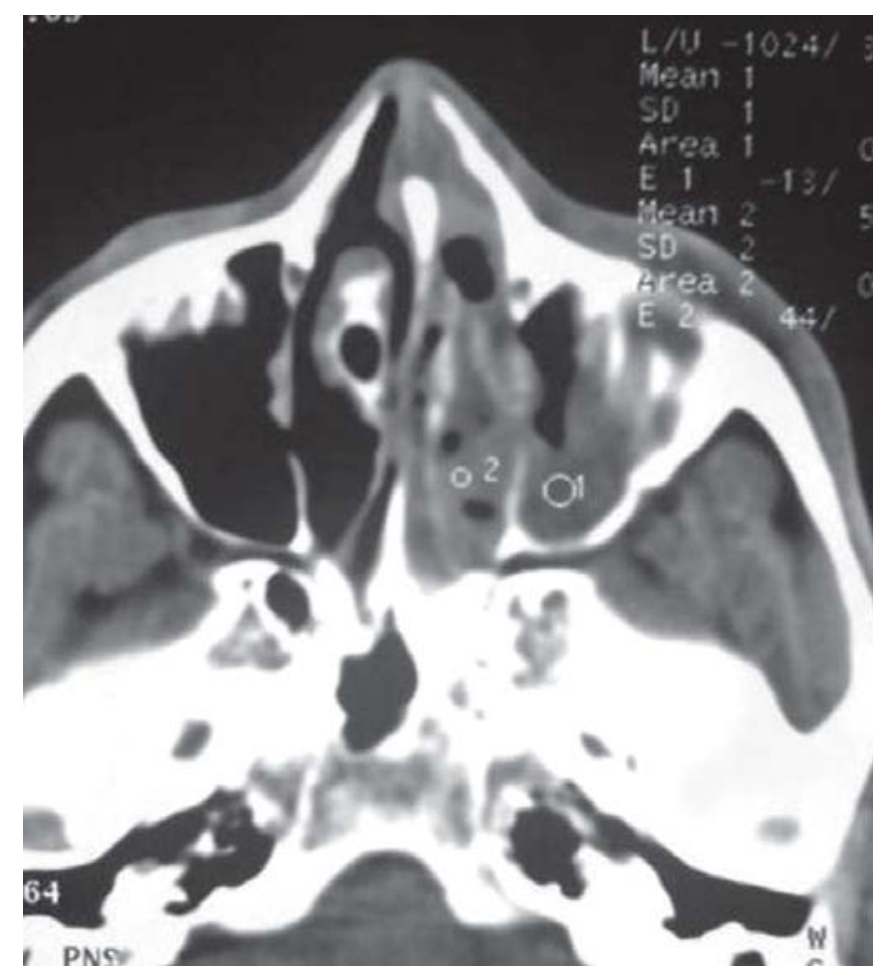

Figure 2: Axial CT scan picture of invasive fungal sinusitis 


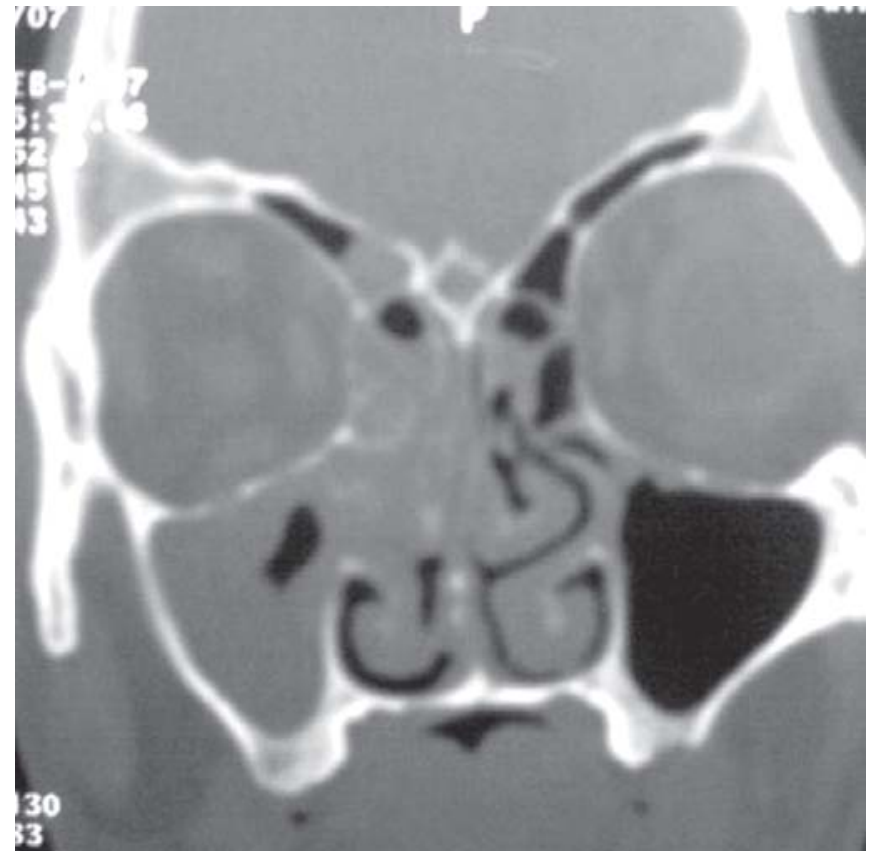

Figure 3: Coronal CT picture of invasive fungal sinusitis

\section{OVERALL RESULTS}

\section{Treatment}

\begin{tabular}{lccl}
\hline Fungus & $\begin{array}{l}\text { Surgical } \\
\text { debridement }\end{array}$ & $\begin{array}{l}\text { I/V } \\
\text { Amphotericin B }\end{array}$ & $\begin{array}{l}\text { Oral } \\
\text { Voriconazole }\end{array}$ \\
\hline Mucormycosis & $20(55 \%)$ & $20(55 \%)$ & $5(14 \%)$ \\
Aspergillus & $16(44 \%)$ & $8(22 \%)$ & $8(22 \%)$ \\
\hline
\end{tabular}

\section{Outcome}

\begin{tabular}{lcc}
\hline & $\begin{array}{c}\text { Surgical debridement } \\
\text { with Amphotericin B }\end{array}$ & $\begin{array}{c}\text { Surgical debridement } \\
\text { with Voriconazole/ } \\
\text { Itraconazole }\end{array}$ \\
\hline Cured & 18 & 8 \\
Persistent disease & 4 & 1 \\
Relapse & 3 & 2 \\
Lost F/U & 1 & 1 \\
Death & 2 & 1 \\
\hline
\end{tabular}

Involvement of ethmoid sinus (70\%) was most common followed by maxillary (68\%), sphenoid (58\%) and frontal (17\%).Unilateral sinus involvement was seen in thirty two patients while four patients had bilateral involvement.

Twenty eight (78\%) patients were treated with IV Amphotericin B with regular monitoring of RFTs, responded well to treatment. Out of which twenty cases were of mucormycosis (Figs 4 and 5) and eight cases were of aspergillus. Five out of these twenty eight cases received

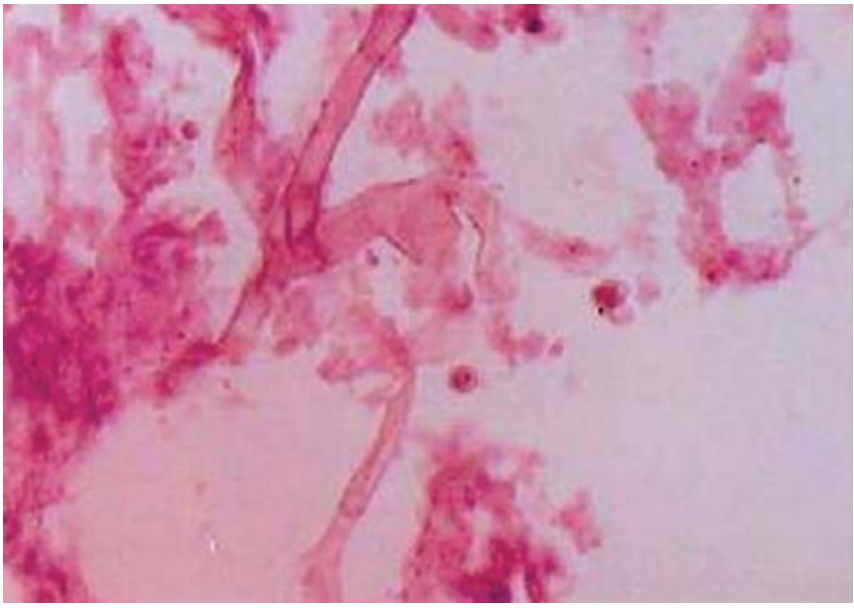

Figure 4: Histopathological picture of mucormycosis

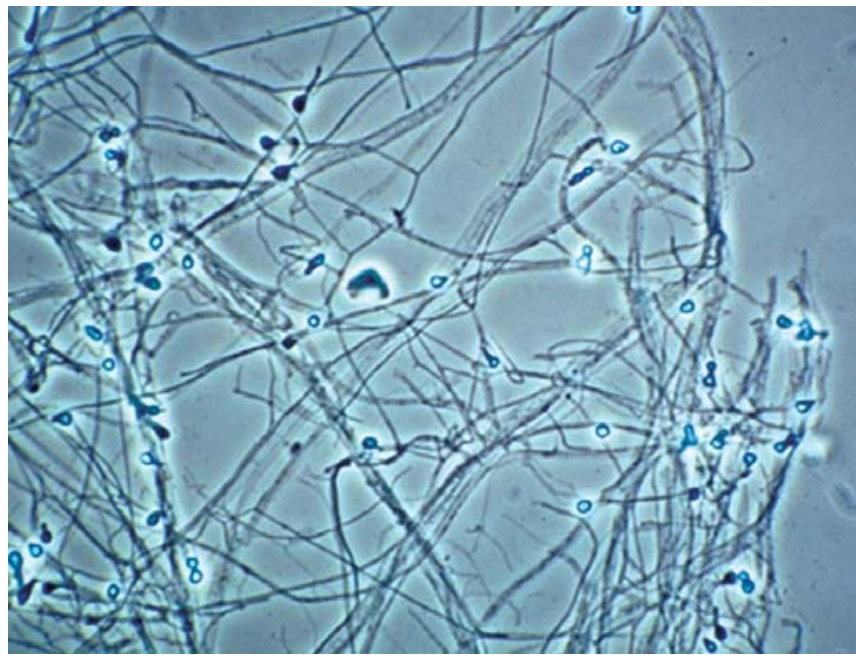

Figure 5: Histopathological picture of mucormycosis

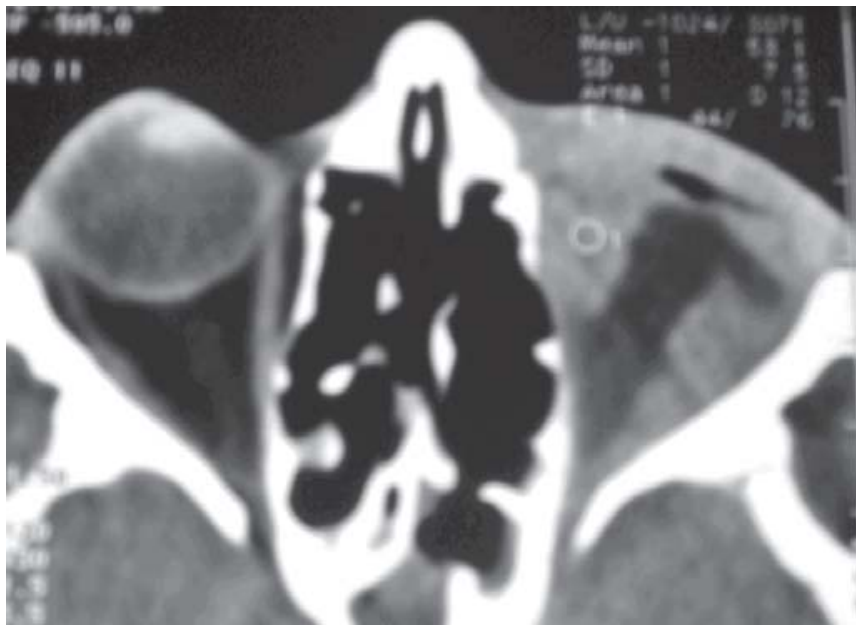

Figure 6: Axial CT scan picture of patient after enucleation

amphotericin followed by voriconazole during follow-up. Rest of the eight cases received Voriconazole only. Four of the patients underwent orbital exenteration (Fig. 6) 
responded well to Amphotericin as compared to other who have not undergone orbital exenteration. Nasal endoscopy was done after monthly interval for six months. Recurrence was seen in only five patients. Two patients were lost during follow-up. Three patients died during the treatment due to CNS involvement and uncontrolled blood sugar levels.

\section{DISCUSSION}

Although the invasive form of fungal sinusitis is uncommon but it is commonly seen to infest immunocompromised patients (McGill, Simpson and Healy, 1980). ${ }^{1}$ It behaves like a malignant neoplasm as described by Hora (1965) ${ }^{2}$ and spreads to adjacent structures such as soft tissues of cheek and orbit. Intracranial extension is fatal in one-fifth of patients even with treatment.

In our study majority of the patients were male in the age group of 36-70 years (64\%). Choi et al has reported female predominance in the age group between 45 -83 years.

Orbital invasive aspergillosis is rare but often fatal. Aspergillosis the most common cause of paranasal sinus mycoses. ${ }^{3}$ Invasive aspergillosis can be either localized or fulminant. Localized disease often starts in the sinuses and spreads to adjacent structures through focal bony erosion or even through vessel walls. The fulminant form is characterized by multiple organ involvement. ${ }^{4}$ Invasive aspergillosis is well documented in immunocompromised patients, with the primary risk factors being neutrophil defects and corticosteroid use. ${ }^{4}$ Other predisposing factors include HIV infection, diabetes mellitus, use of prosthetic devices or trauma, excessive environmental exposure, and possibly advanced age. ${ }^{4}$ In our study, 55\% of patients had diabetes mellitus.

Management often combines surgical debridement along with systemic antifungal drug therapy. Antifungals are used, such as polyenes (Amphotericin) and azoles (Itraconazole and Voriconazole), Among them, Amphotericin B is a conventional drug for treatment of invasive fungal rhinosinusitis. ${ }^{5}$ However, treatment is often prolonged and can be complicated by adverse effects. The most serious complication is renal dysfunction. Newer formulations, including lipid complex and liposomal forms, have been developed to decrease the toxicity of Amphotericin B and indeed seem to be less toxic. ${ }^{6}$ In patients with invasive aspergillosis, initial therapy with Voriconazole led to better responses and improved survival and also resulted in fewer severe side effects than the standard Amphotericin B initial therapy. ${ }^{7}$ In our study patients who were given Voriconazole postsurgical debridement $62 \%$ showed marked improvement. Of the azole class, Itraconazole and Voriconazole are promising and are safer and easier to administer than Amphotericin B. Most experts recommend the maximum daily dose of the chosen antifungal agent(s) until the disease is controlled, and then prolonged administration of oral Itraconazole to ensure eradication thereafter. $^{5}$

Sivak-Callcott et al reported that factors associated with poor prognosis were delayed diagnosis, intracranial extension of infection, and histopathology demonstrating hyphal invasion in blood vessels or adjacent tissue. Debridement of infected and devitalized tissue is necessary because the fungus thrives in necrotic tissue. Early detection and proper treatment may reduce the mortality associated with these diseases. Orbital invasive aspergillosis is often fatal. Risk factors such as fever and incorrect initial diagnosis were found to be associated with high mortality rates in patients with orbital invasive aspergillosis. In those patients without diabetes, survival was also improved; 0 to $47 \%$. The combination of surgery and Amphotericin B resulted in an overall survival rate of $81 \%$.

There is controversy over whether orbital exenteration should be performed, which is cosmetically deforming but it improves the survival rate of patients. Blitzer et al and Ochi et al demonstrated a 78\% survival rate with radical debridement versus $57.5 \%$ with only medical therapy. In our study all the patients who underwent surgical debridement followed by medical treatment showed $72 \%$ cure rate. Amphotericin B is the drug of choice, increasing the survival rate in diabetics from 37 to $79 \%$.

\section{CONCLUSION}

Invasive fungal sinusitis is life threatening in patients with associated immunocompromised state with easy access to brain. Therefore patients with invasive fungal rhinosinusitis with loss of vision should be aggressively managed with orbital exenteration along with antifungals with strict follow-up.

\section{REFERENCES}

1. McGill T J, Simpson G, Healy G B. Fulminant aspergillosis of the nose and paranasal sinuses: A new clinical entity. Laryngoscope 1980;90:748-54.

2. Hora J F. Primary aspergillosis of the nose and paranasal sinuses and associated areas. Laryngoscope 1965;75:768-73. 
3. Chakrabarti A, Sharma SC, Chandler J. Epidemiology and pathogenesis of paranasal sinus mycoses. Otolaryngol Head Neck Surg 1992;107:745-50.

4. Levin LA, Avery R, Shore JW, et al. The spectrum of orbital aspergillosis: A clinicopathological review. Surv Ophthalmol 1996;41:142-54.

5. Stevens DA, Kan VL, Judson MA, et al. Practice guidelines for diseases caused by Aspergillus. Infectious Diseases Society of America. Clin Infect Dis 2000;30:696-709.
6. Walsh TJ, Finberg RW, Arndt C, et al. Liposomal amphotericin B for empirical therapy in patients with persistent fever and neutropenia.National Institute of Allergy and Infectious Diseases Mycoses Study Group. N Engl J Med 1999;340:764-71.

7. Herbrecht R, Denning DW, Patterson TF, et al. Voriconazole versus amphotericin B for primary therapy of invasive aspergillosis, n Engl J Med 2002;347;408-15. 